\title{
Local CD34-positive capillaries decrease in mouse models of kidney disease associating with the severity of glomerular and tubulointerstitial lesions
}

Md Abdul Masum¹, Osamu Ichii', Yaser Hosny Ali Elewa ${ }^{1,2}$, Teppei Nakamura ${ }^{1,3}$ and Yasuhiro Kon ${ }^{1 *}$

\begin{abstract}
Background: The renal vasculature plays important roles in both homeostasis and pathology. In this study, we examined pathological changes in the renal microvascular in mouse models of kidney diseases.

Methods: Glomerular lesions (GLs) in autoimmune disease-prone male BXSB/MpJ-Yaa (Yaa) mice and tubulointerstitial lesions (TILs) in male C57BL/6 mice subjected to unilateral ureteral obstruction (UUO) for 7 days were studied. Collected kidneys were examined using histopathological techniques. A nonparametric Mann-Whitney $U$ test $(P<0.05)$ was performed to compare healthy controls and the experimental mice. The Kruskal-Wallis test was used to compare three or more groups, and multiple comparisons were performed using Scheffe's method when significant differences were observed $(P<0.05)$.

Results: Yaa mice developed severe autoimmune glomerulonephritis, and the number of CD34 ${ }^{+}$glomerular capillaries decreased significantly in GLs compared to that in control mice. However, UUO-treated mice showed severe TILs only, and CD34 $4^{+}$tubulointerstitial capillaries were decreased significantly in TILs with the progression of tubulointerstitial fibrosis compared to those in untreated control kidneys. Infiltrations of B-cells, T-cells, and macrophages increased significantly in the respective lesions of both disease models $(P<0.05)$. In observations of vascular corrosion casts by scanning electron microscopy and of microfil rubber-perfused thick kidney sections by fluorescence microscopy, segmental absences of capillaries were observed in the GLs and TILs of Yaa and UUO-treated mice, respectively. Further, transmission electron microscopy revealed capillary endothelial injury in the respective lesions of both models. The numbers of CD34 ${ }^{+}$ glomerular and tubulointerstitial capillaries were negatively correlated with all examined parameters in GLS $(P<0.05)$ and TILs $(P<0.01)$, respectively.
\end{abstract}

Conclusions: From the analysis of mouse models, we identified inverse pathological correlations between the number of local capillaries in GLs and TILs and the severity of kidney diseases.

Keywords: CD34, Capillary, Kidney disease, Glomerular lesion, Tubulointerstitial lesion

\footnotetext{
* Correspondence: y-kon@vetmed.hokudai.ac.jp

${ }^{1}$ Laboratory of Anatomy, Graduate School of Veterinary Medicine, Hokkaido

University, Kita 18, Nishi 9, Kita-ku, Sapporo, Japan

Full list of author information is available at the end of the article
} 


\section{Background}

Kidney disease causes a systemic deterioration in health. Chronic kidney disease, in particular, is a substantial public health burden because it is associated with end stage renal disease and cardiovascular disease [1]. Various intrinsic and extrinsic factors, such as genomic factors, infections, and drug use, affect the progression of kidney diseases. The kidney is a highly vascularized organ, renal vasculature is also believed to play important roles in both renal function and tissue homeostasis. Altered vasculature can cause several renal injuries mediated by hypoxia-associated processes [2-4]. Local hypoxia in the kidney also results in tubular atrophy, inflammation, and interstitial accumulation of extracellular matrix $[3,4]$. Loss of vascular endothelial growth factor (VEGF) and chronic hypoxia lead to microvascular dysfunction in kidneys [3-6].

The mammalian glomerulus has a well-developed capillary tuft, and these capillaries are lined by thin fenestrated endothelial cells and podocytes that play critical coordinating roles in renal physiology as well as in innate and adaptive immunity [7, 8]. An imbalance in the decrease in endothelial repair and increase in endothelial apoptosis-after renal injury causes glomerular lesions (GLs), such as the loss of the glomerular capillaries and glomerulosclerosis [9]. In diabetic nephropathy, increased blood glucose levels cause capillary injuries [10]. Furthermore, tubulointerstitial capillaries are involved in the regulation of renal function and hemodynamics [2]. Capillary endothelial cells have a crucial function in maintaining renal homeostasis and expressing specific chemokines that control compartment-specific T-cell and monocyte recruitment during inflammation $[11,12]$. This functional variability in endothelial cells is probably associated with differential susceptibilities to apoptosis and differential responses to microenvironmental changes or stimuli. Tubulointerstitial capillaries are also injured in diabetic nephropathy, resulting in a reduction in capillary density and progression of tubulointerstitial lesions (TILs) [13].

Thus, we can distinguish between the renal pathogenesis of GLs and TLs. Earlier drug-induced or spontaneous rodent models, and autoimmune disease models such as MRL/MpJ-Fas ${ }^{\text {lpr/lpr }}$ and BXSB/MpJ-Yaa (Yaa), in particular, have been widely used, and they manifest severe glomerulonephritis caused by immune-complex deposition [14, 15]. Male Yaa mice show more severe glomerulonephritis than females because of a Y-linked autoimmune acceleration (Yaa) mutation on the $\mathrm{Y}$ chromosome; however, we previously clarified that the $\mathrm{BXSB} / \mathrm{MpJ}$ (BXSB)-genetic background also contributes to the progression of autoimmune disease-mediated glomerular damage [14]. In Yaa mice, GLs appeared earlier and were more severe than TILs, which appeared at a late stage in glomerulonephritis [16]. For TILs, unilateral ureteral obstruction (UUO) is usually performed to induce tubulointerstitial fibrosis in mice. UUO results in hydronephrosis, interstitial infiltration of inflammatory cells, tubular cell death from hypoxia, and collagen deposition in the tubulointerstitium, followed by the development of tubulointerstitial fibrosis [17]. A previous study identified TILs, rather than GLs, as the main cause of progressive and end stage kidney disease [18]. However, other researchers have shown the involvement of microvasculature in the advance of human and experimental animal glomerulonephritis [19-21].

Nevertheless, there are no reports clarifying the correlations between altered capillary structures and renal histopathology or renal function in GLs and TILs. The present study investigated the morphological, quantitative, and ultrastructural alterations in glomerular and tubulointerstitial capillaries using two mouse models, namely the spontaneous glomerulonephritis model and UUO model. In addition, based on an analysis of these models, we clarified the correlations between the number of local capillaries in GLs and TILs and kidney diseases severity.

\section{Methods}

Animals

The authors adhered to the Guide for the Care and Use of Laboratory Animals of Hokkaido University, Graduate School of Veterinary Medicine (approved by the Association for Assessment and Accreditation of Laboratory Animal Care International). Animal experimentation was approved by the Institutional Animal Care and Use Committee of the Graduate School of Veterinary Medicine, Hokkaido University (approval no. 13-0032, 16-0124). Experimental mice were purchased from Japan SLC Inc. (Shizuka, Japan). A maximum of five mice were held in one cage containing wood chip bedding material in a pathogen-free animal house. Food and water are provided ad libitum to the animals. Light and dark conditions were maintained at 1:1 ratio. Strain and number of mice used in different disease models and protocols are shown details in Additional file 1. Six month-old male Yaa mice $(N=12)$ were used for the GL model, and same-aged male BXSB mice $(N=12)$ were used as healthy controls. To create the TIL model, 8week-old male C57BL/6 mice $(N=12)$ were subjected to UUO for 7 days. The kidney paired with the UUO kidney in the same mouse was used as a normal control. Briefly, mice were deeply anesthesized with a mixture of $0.3 \mathrm{mg} / \mathrm{kg}$ medetomidine (Kyoritsu Seiyaku, Japan), $4 \mathrm{mg} / \mathrm{kg}$ midazolam (Astellas Pharma, Japan), and $5 \mathrm{mg} / \mathrm{kg}$ butorphanol (Meiji Seika Pharma, Japan), and laparotomy in the sublumbar region was performed to ligate the right ureter tightly with silk thread at the renal hilus. 
Buphrenorphine hydrochloride (Otsuka Pharmaceuticals, Japan) was injected intraperitoneally at a dose rate of $0.3 \mathrm{mg} / \mathrm{kg}$ as an analgesic. Recovery from anesthesia was facilitated by intraperitoneal administration of $0.3 \mathrm{mg} / \mathrm{kg}$ atipamezole (Zenoaq, Japan).

\section{Sample preparation}

Mice without external abnormalities were used in this experiment. The average weights of GLs and TILs model were 25.02 and $20.60 \mathrm{~g}$, respectively. Urine was collected from anesthetized mice. Mice were euthanized by exsanguination from the carotid artery, and blood and kidneys were collected for serological analysis and histological examination, respectively. The kidneys were fixed with neutral buffer formalin (NBF), 4\% paraformaldehyde (PFA) in $0.1 \mathrm{M}$ phosphate buffer (PB, pH 7.4) or $2.5 \%$ glutaraldehyde (GTA) in 0.1 M PB.

\section{Serological and urinary examination}

Serum levels of antibody against anti-double stranded DNA (dsDNA ab) were measured with a Mouse AntidsDNA Ig's (Total A + G + M) ELISA Kit (Alpha Diagnostic International, San Antonio, TX, USA). Serum blood urea nitrogen ( $\mathrm{sBUN}$ ) and creatinine $(\mathrm{sCr})$ levels in all mice were measured using a Fuji Drichem 7000v (Fujifilm, Tokyo, Japan). Urinary albumin creatinine ratios (uACR) were determined using Albuwell $M$ and Creatinine Companion Kits (Exocell, Philadelphia, PA, USA).

\section{Histopathological examination}

Paraffin sections of kidneys fixed with NBF or PFA were cut at a thickness of $2 \mu \mathrm{m}$ and stained with periodic acid Schiff-hematoxylin (PAS-H) or Masson's trichrome (MT). Immunostaining for alpha smooth muscle actin ( $\alpha \mathrm{SMA})$, B220, CD3, CD34, Iba1, and interleukin 1 family, member 6 (IL-1F6/IL-36 $\alpha$ ) [22] was performed to detect myofibroblasts, B-cells, T-cells, capillary endothelial cells, macrophages, and damaged renal tubules, respectively. Staining conditions are listed in Table 1. Briefly, after deparaffinization, kidney sections were subjected to antigen retrieval.
Then, slides were submerged in methanol containing 3\% $\mathrm{H}_{2} \mathrm{O}_{2}$ for $20 \mathrm{~min}$ at room temperature. After blocking, sections were incubated with primary antibody overnight at $4{ }^{\circ} \mathrm{C}$. After washing in phosphate-buffered saline (PBS), sections were incubated with secondary antibody for $30 \mathrm{~min}$ at room temperature, then washed and incubated with streptavidin-biotin complex (SABPO kit, Nichirei, Tokyo, Japan) for $30 \mathrm{~min}$. All sections were then incubated with 3,3-diaminobenzidine tetrahydrochloride- $\mathrm{H}_{2} \mathrm{O}_{2}$ solution. Finally, the sections were counterstained with hematoxylin and dehydrated with an ascending series of alcohols.

\section{Histoplanimetry}

Digital images of over 30 glomeruli or over 30 tubulointerstitial areas randomly selected from each mouse were obtained at 400× magnification using an All-in-One Fluorescence Microscope BZ-X710 (Keyence, Osaka, Japan). The size and number of total cells in each glomerulus were determined using PAS-stained sections. The number of ${\mathrm{B} 220^{+}}^{+}$B-cells, $\mathrm{CD}^{+}$T-cells, $\mathrm{Iba}^{+}$macrophages, and $\mathrm{CD}_{3} 4^{+}$capillaries observed in the digital images of glomeruli were counted using immunohistochemical sections and a BZ-X Analyzer (Keyence). Further, glomerular damage was semi-quantitatively scored according to methods described by Ichii et al. [23]. For TILs, the numbers of $\mathrm{B}^{2} 20^{+}$B-cells, CD3 ${ }^{+}$T-cells and IL-1F6/IL-36 $\alpha^{+}$ damaged tubules throughout the cortex were counted. Additionally, $\mathrm{CD}_{3} 4^{+}$capillaries, $\mathrm{Iba}^{+}$macrophages, and ${\alpha \mathrm{SMA}^{+}}^{+}$reaction areas in the tubulointerstitium were counted using immunohistochemical sections and a BZ-X Analyzer (Keyence), based on digital images of the renal cortex.

\section{Ultrastructural examination}

Kidneys were pre-fixed with $2.5 \%$ GTA in $0.1 \mathrm{M}$ PB for $4 \mathrm{~h}$ at $4{ }^{\circ} \mathrm{C}$, post-fixed with $1 \%$ osmium tetroxide in $0.1 \mathrm{M} \mathrm{PB}$ for $2 \mathrm{~h}$ at $4{ }^{\circ} \mathrm{C}$, and then dehydrated in graded alcohol and embedded in epoxy resin (Quetol 812 mixtures; Nisshin EM, Tokyo, Japan). Ultrathin sections

Table 1 Summary of immunostaining conditions

\begin{tabular}{|c|c|c|c|c|c|c|}
\hline & aSMA & B220 & CD3 & CD34 & Iba1 & IL-1F6/IL-36a \\
\hline $\begin{array}{l}\text { Antigen } \\
\text { retrieval }\end{array}$ & CB $105^{\circ} \mathrm{C}, 20 \mathrm{~min}$ & CB $105^{\circ} \mathrm{C}, 20 \mathrm{~min}$ & $\mathrm{~TB} 105^{\circ} \mathrm{C}, 20 \mathrm{~min}$ & CB $105^{\circ} \mathrm{C}, 20 \mathrm{~min}$ & $\begin{array}{l}0.1 \% \text { pepsin } 37^{\circ} \mathrm{C} \\
5 \mathrm{~min}\end{array}$ & $\mathrm{CB} 105^{\circ} \mathrm{C}, 20 \mathrm{~min}$ \\
\hline Blocking & $10 \%$ NGS & $10 \%$ NGS & $10 \%$ NGS & $10 \%$ NGS & $10 \%$ NGS & $5 \%$ NDS \\
\hline $\begin{array}{l}\text { Primary } \\
\text { antibody }\end{array}$ & $\begin{array}{l}\text { Rabbit polyclonal } \\
\text { antibodies (Abcam, } \\
\text { Cambridge, UK) } \\
\text { 1:3000 }\end{array}$ & $\begin{array}{l}\text { Rat polyclonal } \\
\text { antibodies (Cedarlane, } \\
\text { Ontario, Canada) 1:1000 }\end{array}$ & $\begin{array}{l}\text { Rabbit polyclonal } \\
\text { antibodies (Nichirei, } \\
\text { Tokyo, Japan) 1:200 }\end{array}$ & $\begin{array}{l}\text { Rabbit polyclonal } \\
\text { antibodies (Abcam, } \\
\text { Cambridge, UK) 1:400 }\end{array}$ & $\begin{array}{l}\text { Rabbit polyclonal } \\
\text { antibodies (Wako, } \\
\text { Tokyo, Japan) 1:2000 }\end{array}$ & $\begin{array}{l}\text { Goat polyclonal } \\
\text { antibodies (R\&D } \\
\text { Systems, Minnesota, } \\
\text { USA) 1:400 }\end{array}$ \\
\hline $\begin{array}{l}\text { Biotinylated } \\
\text { secondary } \\
\text { antibody }\end{array}$ & $\begin{array}{l}\text { Goat anti-rabbit } \\
\text { (SABPO kit, Nichirei, } \\
\text { Tokyo, Japan) } \\
\text { 1:100 }\end{array}$ & $\begin{array}{l}\text { Rat anti-goat lgG (Caltag } \\
\text { Medsystems, Buckingham, } \\
\text { UK) 1:100 }\end{array}$ & $\begin{array}{l}\text { Goat anti-rabbit } \\
\text { (SABPO kit, Nichirei, } \\
\text { Tokyo, Japan) 1:100 }\end{array}$ & $\begin{array}{l}\text { Rat anti-goat IgG } \\
\text { (Caltag Medsystems, } \\
\text { Buckingham, UK) } \\
\text { 1:100 }\end{array}$ & $\begin{array}{l}\text { Goat anti-rabbit lgG } \\
\text { (SABPO kit, Nichirei, } \\
\text { Tokyo, Japan) 1:100 }\end{array}$ & $\begin{array}{l}\text { Donkey anti-goat } \\
\text { lgG (Santa Cruz, } \\
\text { California, USA) } \\
\text { 1:100 }\end{array}$ \\
\hline
\end{tabular}


$(60 \mathrm{~nm})$ were stained with uranyl acetate and lead citrate. Mounted samples were observed under a JEOL transmission electron microscope (TEM, JEM-1210; JEOL, Tokyo, Japan).

\section{Visualization of the renal vasculature}

Vascular corrosion casts of kidney were prepared according a method described by Verli et al. [24]. Mice other than those used for the histological analysis $(N=4)$ were used for vascular casts of mice in both the control and disease groups. Briefly, after euthanasia, PBS containing EDTA, followed by a mixture of resin and catalyst (Mercox II cast, Ladd Research, Williston, USA) was perfused through left ventricles of heart. Dissected kidneys were held in a water bath at $37^{\circ} \mathrm{C}$ overnight to allow polymerization with resin. Tissue surrounding the vascular bed was corroded by applying $15 \%$ potassium hydroxide for $30 \mathrm{~h}$ at room temperature. After washing with distilled water, casts that emerged in $t$-butyl alcohol were subjected to freeze-drying in a vacuum freeze dryer (ES-2030, Hitachi, Tokyo, Japan). The dried specimens were mounted on a specimen stub, sputter-coated using a Hitachi E-1030 ion sputter coater (Hitachi, Tokyo, Japan), and then examined by scanning electron microscopy (SEM, S-4100; Hitachi, Tokyo, Japan) with an accelerating voltage of $4 \mathrm{kV}$.

In addition, after euthanasia, rubber (Microfil, Flow Tech, Inc. Massachusetts, USA) was perfused through left ventricles according to a method described by Walker et al. [25]. Then, dissected kidneys were fixed with $4 \%$ PFA in $0.1 \mathrm{M} \mathrm{PB}$ overnight at $4{ }^{\circ} \mathrm{C}$. Fixed kidneys were cut into 200 - $\mu \mathrm{m}$-thick sections using a microslicer (DSK Microslicer DTK-3000, Ted Pella, Inc. Redding, USA) and hydrated with an ascending series of alcohols. Finally, thick sections were cleared with methyl salicylate and examined under a BZ-X Analyzer of an All-in-One Fluorescence Microscope BZ-X710 (Keyence, Osaka, Japan) to obtain Z-stack images.

\section{Statistical analysis}

Results are expressed as the mean \pm standard error (SE). For comparisons between healthy controls and experimental mice, a nonparametric Mann-Whitney $U$ test $(P<0.05)$ was utilized. The Kruskal-Wallis test was used to compare three or more populations, and multiple comparisons were performed using Scheffe's method when significant differences were observed $(P<0.05)$. Correlations between the $\mathrm{CD}_{3} 4^{+}$renal capillary number and renal function or histopathological indices were analyzed using Spearman's rank correlation coefficient $(P<0.05)$.

\section{Results}

Histopathological features of GL and TIL models

In the GL model, Yaa mice clearly developed membranoproliferative glomerulonephritis features characterized by increased glomerular cell numbers and glomerular sizes, thickening of glomerular basement membranes, PAS-positive deposits, and adhesion of podocytes to parietal cells, whereas control BXSB mice did not (Fig. 1a and Additional file 2). No obvious GLs were observed in the TIL model, but UUO kidneys showed severe TILs characterized by dilated tubules with urinary casts, dilation of renal tubular lumens, and immune cell infiltrations in the tubulointerstitium (Fig. 1a). By immunohistochemistry (Fig. 1b-d), numerous $\mathrm{B}^{2} 20^{+}$Bcells, $\mathrm{CD}^{+} \mathrm{T}$-cells, and $\mathrm{Iba1}^{+}$macrophages were observed in the glomeruli of Yaa mice; however, few cells were found in the BXSB and TIL mice. In UUO kidneys, a few B-cells (Fig. 1b) and numerous T-cells and macrophages (Fig. 1c and d) were observed in TILs, relative to those in the control kidneys. With MT staining (Fig. 1e), aniline blue-positive sclerotic and fibrotic lesions were observed in GLs of Yaa mice and TILs of UUO kidneys, respectively, but these were not found in their respective controls.

CD34 immunostaining revealed positive reactions in the capillary endothelium of glomerulus and in the tubulointerstitium (Fig. 1f). However, in the glomeruli of Yaa mice, $\mathrm{CD}_{34}{ }^{+}$staining was faint, although a few capillaries were observed at the peripheral regions of glomeruli. The localizations and intensities of $\mathrm{CD} 34^{+}$capillaries in the tubulointerstitium were comparable between Yaa and BXSB mice. However, UUO kidneys showed a decreased number and intensity of $\mathrm{CD}^{+} 4^{+}$reactions in TILs. Interestingly, CD34- capillaries, capillaries with lumens that showed no $\mathrm{CD} 34^{+}$staining, were also found in UUO kidneys. CD34 ${ }^{+}$capillaries disappeared near damaged renal tubules with the development of fibrosis, although positive staining in the glomerulus and TILs without fibrotic features were evident. In control kidneys, $\mathrm{CD}_{34}{ }^{+}$capillaries were observed clearly in both the glomerulus and tubulointerstitium.

\section{Quantitative evaluations of renal histopathological changes in GL and TIL models}

In the GL model mice, indices of renal function and autoimmune disease were determined. Yaa mice showed significantly higher levels of $\mathrm{sBUN}, \mathrm{sCr}, \mathrm{uACR}$, and serum anti-dsDNA abs than BXSB mice (Fig. 2a).

As for GL parameters such as glomerular size, cell number, and damage score, Yaa mice showed significantly higher values than BXSB and TIL mice (Fig. 2b and Additional file 3). A similar tendency was observed in the number of $\mathrm{B}^{2} 20^{+} \mathrm{B}$-cells, $\mathrm{CD}^{+}{ }^{+}$T-cells, and $\mathrm{Iba}^{+}$ macrophages per glomerulus, with Yaa mice showing higher values than BXSB and TIL mice (Fig. 2c and Additional file 3). Importantly, CD34 ${ }^{+}$glomerular capillaries decreased significantly in Yaa mice relative to those in BXSB mice (Fig. 2c and Additional file 3). 


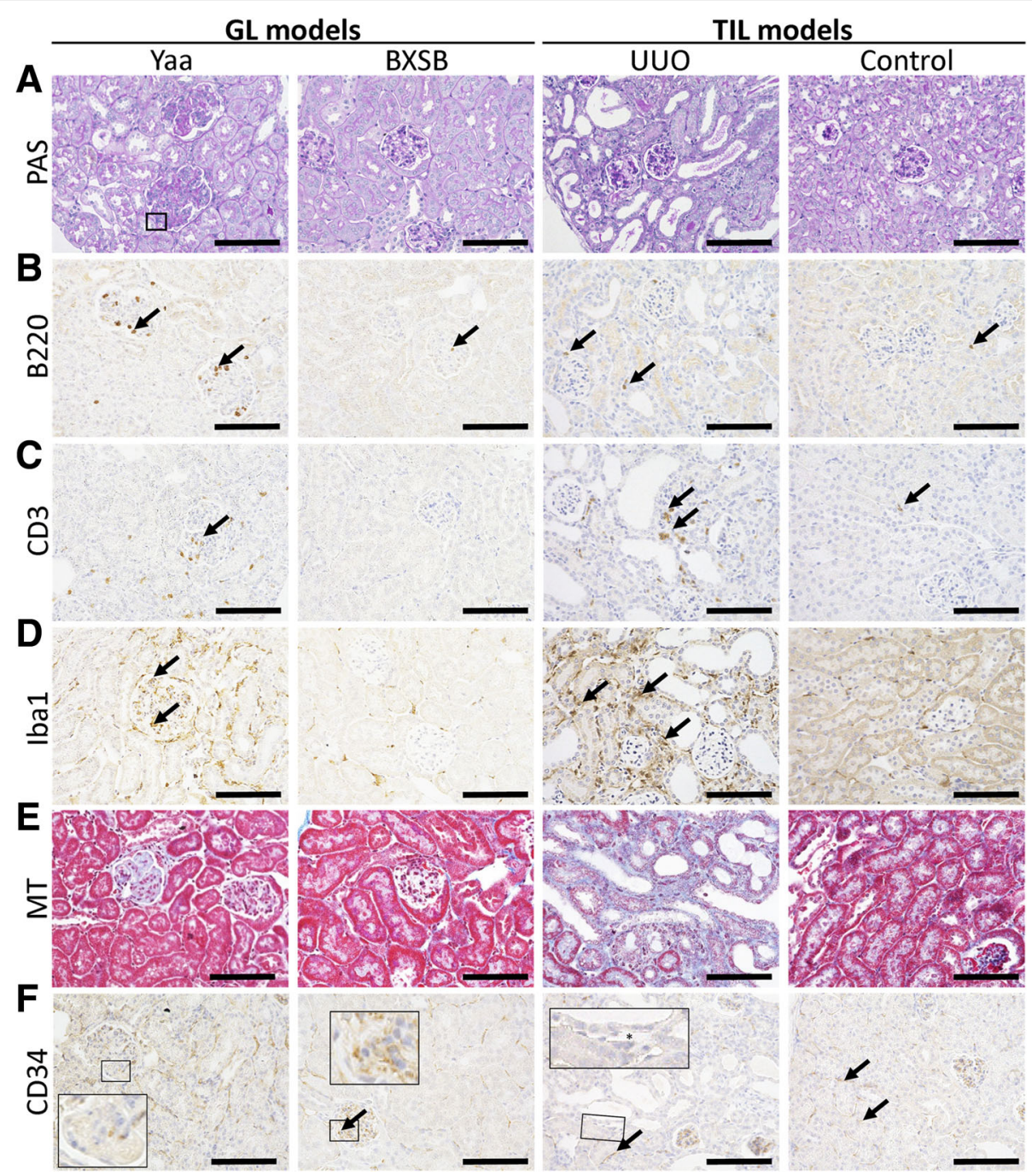

Fig. 1 Glomerular and tubulointerstitial histopathology in glomerular lesion (GL) and tubulointerstitial lesion (TIL) mouse models. a Histopathological features of GLs in Yaa mice (GL model) and TILs in unilateral ureteral obstruction (UUO) mice (TIL model) and, their respective controls. Periodic acid Schiff-hematoxylin (PAS-H) staining. b-d Analysis of B220 B-cell, CD3 ${ }^{+}$T-cells, and Iba- $1^{+}$macrophage infiltration in GLs of Yaa mice and TILs of UUO mice and, their respective controls. Immunohistochemistry. e Evaluations of renal fibrosis in the kidneys of Yaa mice and UUO mice and, their respective controls. MT staining. $\mathbf{f}$ Analysis of CD34 ${ }^{+}$cells in GLs of Yaa mice and TILs of UUO mice and, their respective controls. Immunohistochemistry.

All bars $=100 \mu \mathrm{m}$

As for TIL parameters such as the number of $\mathrm{B}^{2} 20^{+}$ B-cells, $\mathrm{CD}^{+}{ }^{\mathrm{T}}$-cells, $\mathrm{Iba1}^{+}$macrophages, $\alpha \mathrm{SMA}^{+}$ myofibroblasts, and IL-1F6/IL-36 $\alpha^{+}$damaged renal tubules in the renal cortex, UUO kidneys showed significantly higher values than control kidneys (Fig. 3 and Additional file 4). Yaa mice also showed significantly higher values for $\mathrm{B}_{220}{ }^{+} \mathrm{B}$-cells, $\mathrm{CD}^{+}{ }^{+}$T-cells, and IL-1F6/IL- $36 \alpha^{+}$damaged renal tubules than BXSB mice (Fig. 3 and Additional file 4). Further, UUO kidneys showed significantly higher values for $\mathrm{CD}^{+}{ }^{+} \mathrm{T}$-cells, $\mathrm{Iba}^{+}$macrophages, $\alpha \mathrm{SMA}^{+}$myofibroblasts, and IL-1F6/IL-36 $\alpha^{+}$damaged renal tubules than kidneys in GL mice (Fig. 3 and Additional file 4). CD34 ${ }^{+}$ tubulointerstitial capillaries decrease significantly in UUO kidneys compared to others (Fig. 3 and Additional file 4).

\section{Morphological alterations in capillaries of GL and TIL} models

In thick sections of rubber-injected kidneys (Fig. 4a), alterations in both glomerular and tubulointerstitial capillaries were not obvious in Yaa and BXSB mice. However, tubulointerstitial capillary loss was evident in UUO kidneys, in contrast control kidneys showed a fine capillary network. Under SEM, vascular corrosion casts (Fig. 4b) from BXSB mice showed normal tufts of glomerular capillaries, whereas Yaa mice showed segmental loss of glomerular capillaries. Interestingly, segmental loss of tubulointerstitial capillaries was observed in UUO kidneys rather than glomerular capillaries compared to those in control kidneys. 


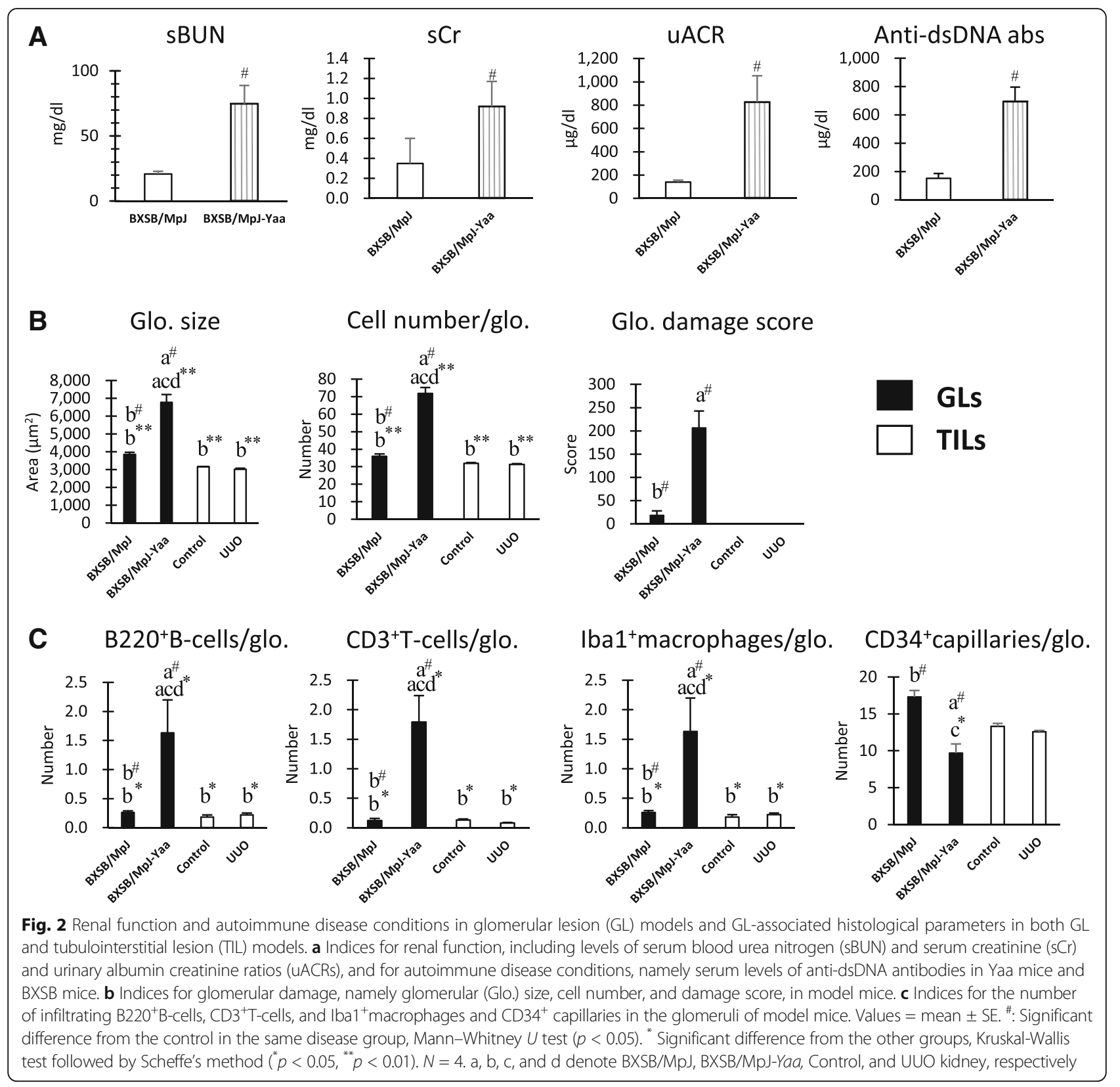

In ultrastructural observation of the GL model mice by TEM, loss of glomerular capillaries, podocyte injury with accumulation of mesangial matrix, and proliferation of mesangial cells were found in glomerulosclerosed areas of glomeruli in Yaa mice (Fig. 5A-a). Glomerular capillaries showed thickened endothelial cytoplasms with vacuolation and thickened capillary basement membranes (Fig. 5A-b). Sub endothelial vacuolation was found just beneath the detaching endothelium (Fig. 5A-c). Capillary basement membranes were thickened and projected into lumens, resulting in partial occlusion of capillary lumens (Fig. 5A-c). Glomerular capillaries at the periphery showed thickened endothelial cytoplasms with loss of endothelial fenestration
(Fig. 5A-d), detached endothelia, and the accumulation of immune cells in capillary lumens (Fig. 5A-e and -f). Podocyte injuries were characterized by foot process effacement in areas adjacent to thickened endothelial layers of capillaries (Fig. 5A-g). Ultrastructure were well preserved in glomerular capillaries and podocytes in BXSB mice (Fig. 5A -h).

In the TIL model, tubulointerstitial capillary injuries were clearly observed in UUO kidneys (Fig. 5B). These injuries were characterized by stratification of thickened endothelial cytoplasms with vacuolation, and wide subendothelial spaces were also found in tubulointerstitial capillaries (Fig. 5B-a). Endothelia detaching from tubulointerstitial capillaries were observed concurrently with 

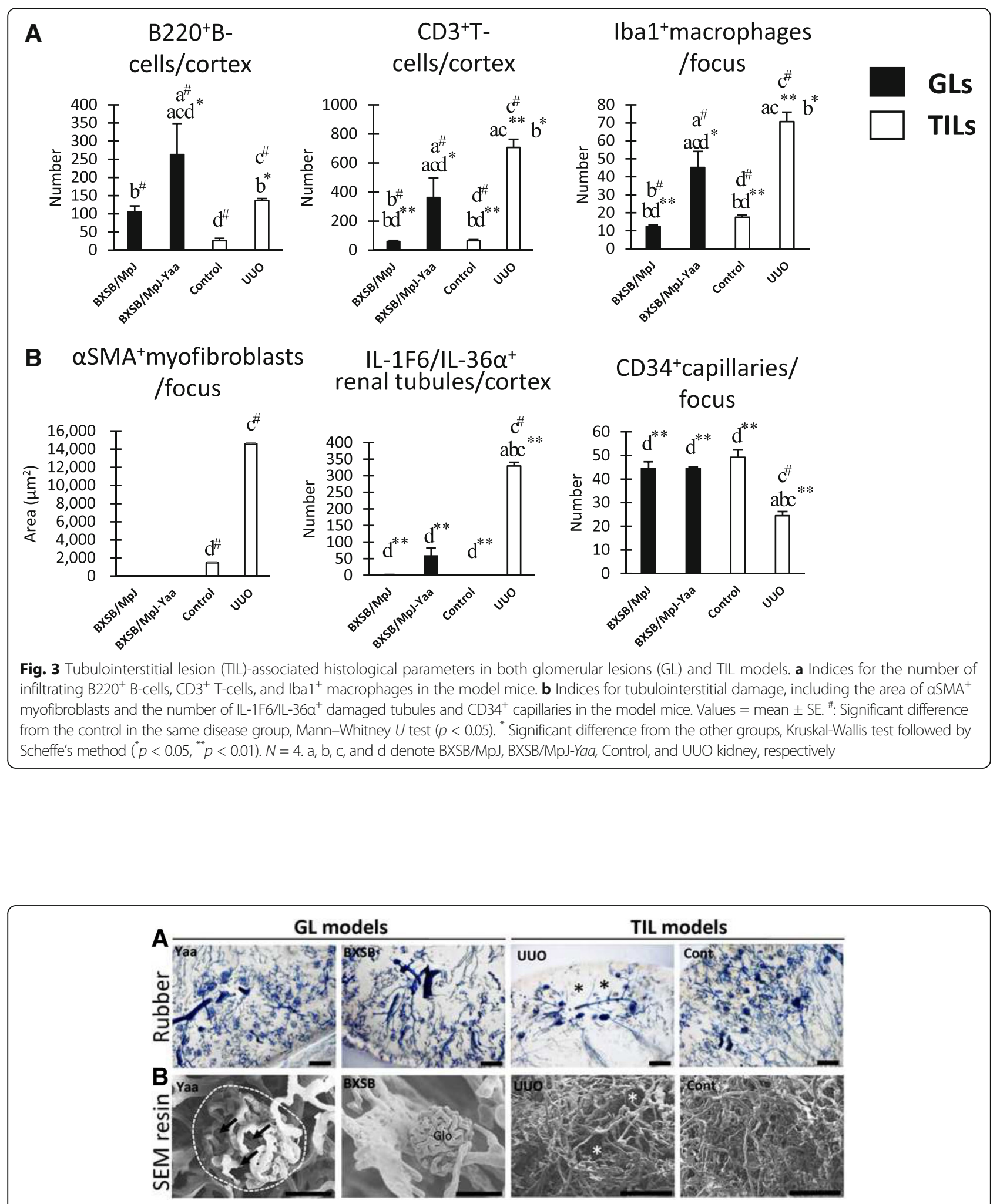

Fig. 4 Segmental loss of capillaries in glomerular lesions (GLs) and tubulointerstitial lesions (TILs). a Capillary structures in the kidneys of Yaa mice, unilateral ureteral obstruction (UUO) mice, and their respective controls, visualized in thick kidney section following by rubber perfusion. Bars $=200 \mu \mathrm{m}$. Asterisks indicate segmental loss of tubulointerstitial capillaries. b Capillary structures in the kidneys of Yaa mice, UUO mice, and their respective controls, visualized by the cast method and scanning electron microscopy. Bars $=50 \mu \mathrm{m}$. Arrows and asterisks indicate segmental loss of glomerular and tubulointerstitial capillaries, respectively 

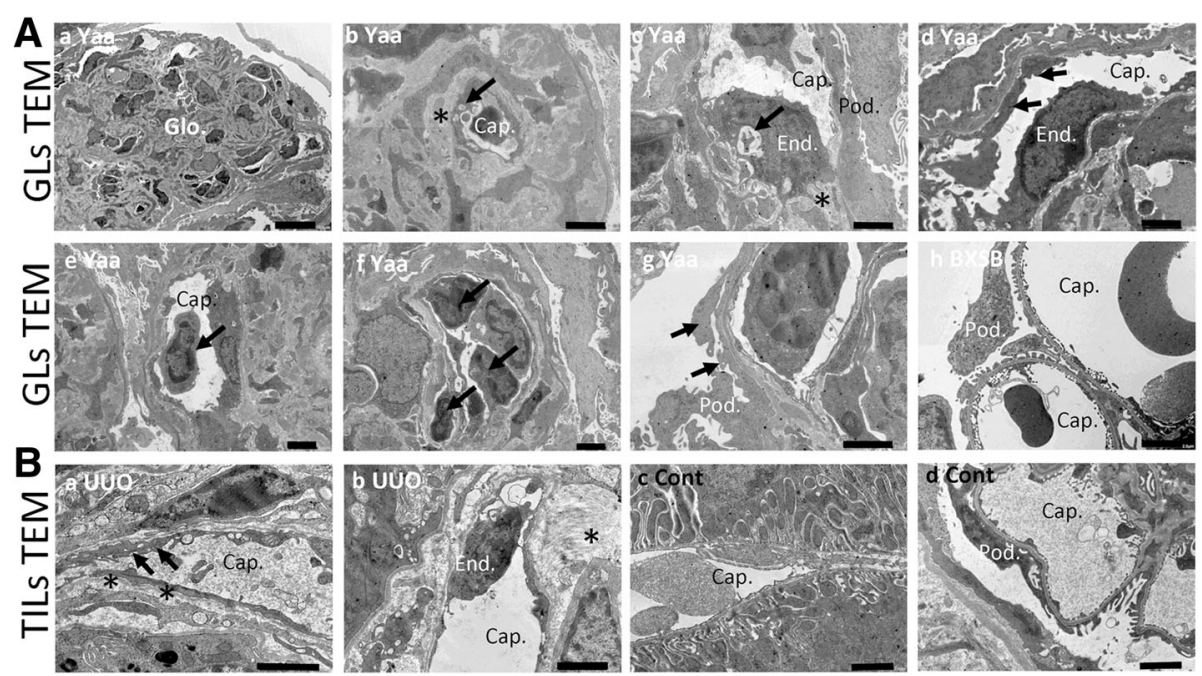

Fig. 5 Ultrastructural alterations in glomerular and tubulointerstitial capillary morphologies in model mice. a Ultrastructures of glomerular capillaries in the GL model by transmission electron microscopy. Injury and loss of glomerular capillaries and podocyte injury with accumulation of increased mesangial cells and matrix in Yaa mice (panel a). Thickened endothelial cytoplasms and basement membranes (asterisk) with vacuolation (arrow) (panel b). Detaching endothelium with subendothelial vacuolation (arrow) and thickened basement membranes projecting into a capillary lumen (asterisk) (panel c). Loss of endothelial fenestration in a peripheral capillary (arrows) (panel d). Detached endothelium and immune cells in the capillary lumen (arrows) (panels e and f). Podocyte foot process effacement (arrows) (panel g). Glomerular capillaries and podocytes ultrastructures were well preserved in BXSB mice (panel h). All bars $=2 \mu \mathrm{m}$, except for panel $\mathbf{a}=10 \mu \mathrm{m}$. $\mathbf{b}$ Ultrastructures of tubulointerstitial capillaries in the TLL model by transmission electron microscopy. Stratification of a thickened endothelial cytoplasm with vacuolation (arrows) and wide subendothelial space (asterisks) (panel a). Endothelium (arrows) detaching from a tubulointerstitial capillary with accumulation of collagen fibers around the capillary (asterisk) (panel b). Control kidneys showed normal tubulointerstitial capillaries and podocytes (panels c and d). All bars $=2 \mu \mathrm{m}$. Cap: capillary. End: endothelial cell. Glo: glomerulus. Pod: podocyte

the accumulation of collagen fibers around capillaries, resulting in narrowing of capillary lumens (Fig. 5B -b). Control kidneys showed normal glomerular and tubulointerstitial capillaries as well as podocyte morphology (Fig. 5B-c and -d).

\section{Correlations between the number of $\mathrm{CD} 34^{+}$capillaries and histopathological indices in GL and TIL models} The number of $\mathrm{CD} 34^{+}$glomerular capillaries in GL mice were significantly and negatively correlated with sBUN, $\mathrm{sCr}$, uACR, serum levels of anti-dsDNA ab, glomerular size, glomerular cell number, glomerular damage score, and infiltrations of $\mathrm{B} 220^{+} \mathrm{B}$-cells, $\mathrm{CD}^{+}{ }^{+}$-cells, and Iba1 + macrophages in glomeruli (Table 2). However, the number of $\mathrm{CD}_{4} 4^{+}$glomerular capillaries in TILs model mice did not correlate with glomerular histopathological parameters in these mice (Table 2). Conversely, the number of $\mathrm{CD}_{3} 4^{+}$tubulointerstitial capillaries was significantly and negatively correlated with the number of infiltrating $\mathrm{B}_{220^{+}} \mathrm{B}_{\text {-cells, }} \mathrm{CD}^{+}{ }^{+} \mathrm{T}$-cells, $\mathrm{Iba}^{+}$macrophages, IL-1F6/IL-36 $\alpha^{+}$damaged renal tubules, and $\alpha \mathrm{SMA}^{+}$myofibroblasts in the TIL (Table 3 ), but only with infiltrating $\mathrm{CD}^{+}{ }^{+} \mathrm{T}$-cells and $\mathrm{Iba}^{+}$macrophages in GL, model (Table 3).

\section{Discussion}

For the GL model using Yaa mice, the number of glomeruli with cells positive for $\mathrm{CD} 34$, a representative

Table 2 Correlation between CD34 $4^{+}$glomerular capillaries and histopathological parameters of glomeruli in GL and TIL model mice

\begin{tabular}{|c|c|c|c|c|c|c|c|c|}
\hline Parameters & & \multicolumn{7}{|c|}{ Indices for glomerular histopathology } \\
\hline \multirow{2}{*}{ GL model } & BXSB/MpJ & $3850 \pm 116.35^{\text {bt }}$ & $35.91 \pm 1.40^{\mathrm{b \#}}$ & $18.25+98^{\mathrm{b \#}}$ & $0.26+0.03^{\mathrm{bH}}$ & $0.12+0.04^{\mathrm{b} \#}$ & $0.13+0.02^{\mathrm{b \#}}$ & $1729+088^{\text {b\# }}$ \\
\hline & BXSB/MpJ-Yaa & $6771.94 \pm 446.32^{\mathrm{a \# acd**}}$ & $71.85 \pm 3.30^{\text {a\#acd**}}$ & $206.25 \pm 36.29^{\mathrm{a \#}}$ & $1.63 \pm 0.57^{\mathrm{a} \# \mathrm{acd} \mathrm{d}^{*}}$ & $1.79 \pm 0.45^{\text {a\#acd* }^{*}}$ & $3.57 \pm 0.40^{\text {a\#acd* }}$ & $9.68 \pm 1.25^{\text {a\#аc* }}$ \\
\hline \multirow[t]{2}{*}{ TIL model } & Control kidney & $3161.13 \pm 0.53^{b^{* *}}$ & $31.88 \pm 0.53^{b^{* *}}$ & 0 & $0.18 \pm 0.04^{\mathrm{b}^{*}}$ & $0.13 \pm 0.02^{\mathrm{b}^{*}}$ & $0.07 \pm 0.01^{b^{*}}$ & $13.3 \pm 0.42^{b^{*}}$ \\
\hline & UUO kidney & $3010 \pm 64.50^{\mathrm{b}^{* *}}$ & $31.31 \pm 0.47^{\mathrm{b}^{* *}}$ & 0 & $0.22 \pm 0.03^{\mathrm{b}^{*}}$ & $0.08 \pm 0.01^{\mathrm{b}^{*}}$ & $0.03 \pm 0.0^{\mathrm{b}^{*}}$ & $12.56 \pm 0.19$ \\
\hline
\end{tabular}

Values = mean \pm SE. " : Significant difference from the control in the same disease group, Mann-Whitney $U$ test $(p<0.05)$. ${ }^{*}$ Significant difference from the other groups, Kruskal-Wallis test followed by Scheffe's method ( $\left.{ }^{*} p<0.05,{ }^{* * *} p<0.01\right) . N=4$. Glo.: glomerular; Pod: podocyte; GLs: glomerular lesions; TILs: tubulointerstitial lesions

$\mathrm{a}, \mathrm{b}, \mathrm{c}$ and ${ }^{\mathrm{d}}$ denotes BXSB/MpJ, BXSB/MpJ-Yaa, Control and UUO kidney, respectively 
Table 3 Correlation of $\mathrm{CD}_{3} 4^{+}$tubulointerstitial capillaries with histopathological parameters of the tubulointerstitium in GL and TIL model mice

\begin{tabular}{|c|c|c|c|c|c|c|c|}
\hline \multirow[t]{2}{*}{ Parameters } & & \multicolumn{6}{|c|}{ Indices for tubulointerstitial histopathology } \\
\hline & & $\begin{array}{l}\text { Ti. B220+B-cells/ } \\
\text { cortex }\end{array}$ & $\begin{array}{l}\text { Ti. CD3 }{ }^{+} \text {T-cells/ } \\
\text { cortex }\end{array}$ & $\begin{array}{l}\text { Ti. Iba }{ }^{+} \\
\text {macrophages/focus }\end{array}$ & $\begin{array}{l}\text { Ti. aSMA } \\
\text { myofibroblasts/focus }\end{array}$ & $\begin{array}{l}\text { IL-1F6/IL-36a }{ }^{+} \text {renal } \\
\text { tubules/focus }\end{array}$ & $\begin{array}{l}\text { CD34 + tubulointerstitial } \\
\text { capillaries/focus }\end{array}$ \\
\hline \multirow[t]{2}{*}{ GL model } & BXSB/MpJ & $104.75 \pm 16.70^{\mathrm{b \#}}$ & $59.75 \pm 7.27^{\text {b\# bd* }}$ & $12.43 \pm 0.88^{\mathrm{b \#} \mathrm{bd*}}$ & 0 & $1.25 \pm 1.25^{\mathrm{d}^{* *}}$ & $44.52 \pm 2.76^{\mathrm{d}^{* *}}$ \\
\hline & BXSB/MpJ-Yaa & $263 \pm 85.90^{\text {a\# acd* }}$ & $362.75 \pm 133.06^{\mathrm{a \#} a c d^{*}}$ & $45.25 \pm 8.91^{\text {a\# acd* }}$ & 0 & $58.5 \pm 24.38^{\mathrm{d}^{* *}}$ & $44.51 \pm 0.60^{\mathrm{d}^{* *}}$ \\
\hline \multirow[t]{2}{*}{ TIL model } & Control kidney & $25.75 \pm 6.90^{\mathrm{d \#}}$ & $65.5 \pm 6.75^{\mathrm{d} \# b d^{*}}$ & $17.51 \pm 1.31^{\mathrm{d} \# \mathrm{bd}^{*}}$ & $1463.06 \pm 39.12^{\mathrm{d \# \#}}$ & 0 & $49.22 \pm 3.12^{\mathrm{d}^{* *}}$ \\
\hline & UUO kidney & $136 \pm 6.14^{\mathrm{c \#} \mathrm{b*}}$ & $707 \pm 55.48^{\mathrm{c \#} a c^{* *} b^{*}}$ & $70.73 \pm 5.20^{\mathrm{c \#} \mathrm{a} c^{* *} b^{*}}$ & $14,579 \pm 78.62^{\mathrm{c \#}}$ & $329.66 \pm 11.01^{\mathrm{c \#} \mathrm{abc}}$ & $24.4 \pm 1.86^{\mathrm{c \#} a b c^{*}}$ \\
\hline
\end{tabular}

Values $=$ mean \pm SE. ${ }^{\#}$ : Significant difference from the control in the same disease group, Mann-Whitney $U$ test $(p<0.05) .{ }^{*}$ Significant difference from the other groups, Kruskal-Wallis test followed by Scheffe's method ( $\left.{ }^{*} p<0.05,{ }^{* *} p<0.01\right) . N=4$. Ti.: tubulointerstitial; GLs: glomerular lesions; TILs: tubulointerstitial lesions a, b, cand denotes BXSB/MpJ, BXSB/MpJ-Yaa, Control and UUO kidney, respectively

marker of endothelial cells, significantly and negatively correlated with serum levels of autoantibody and deterioration in renal function, as well as increased numbers of infiltrating cells in the glomeruli. TILs in GL models showed an increase in cell infiltration and damaged renal tubules, but not myofibroblasts; however, the number of CD34 $^{+}$capillaries in TILs was comparable between BXSB and Yaa mice and did not correlate with any renal pathological parameters. Further, no significant GLs were noted in UUO models. The Yaa mouse is a model of spontaneous autoimmune-mediated membranoproliferative glomerulonephritis, with hyperproliferation of pathogenic B-cells resulting from a Yaa mutation that contributes to autoantibody production, activation of pathogenic T-cells, and secretion of pro-inflammatory cytokines that contribute to the development of GLs [16, 22, 26-28]. Therefore, GL events appear earlier and were more severe than TILs in Yaa mice, whereas severe TILs, with fibrotic features characterized by an increase in myofibroblasts, were prominent end stage kidney disease in Yaa mice. However, because glomerular efferent arterioles directly connect to branches of peritubular/tubulointerstitial capillaries, a reduction in blood supply to the glomerulus because of GLs might affect TILs. Collectively, the study data indicate that a decrease in $\mathrm{CD}_{3} 4^{+}$cells in glomerular capillaries correlates with the progression of GLs in autoimmune disease-prone Yaa mice.

In GL models, glomerular capillaries had narrow lumens, thickened endothelial cytoplasms with vacuolation, loss of endothelial fenestration, and detached luminal endothelia in capillary lumens. Although renal tubulointerstitial capillary injury, particularly capillary basement membrane multilamination, is prominent feature in chronic microvascular injury in renal allograft rejection [29, 30], we did not find such features in the kidneys of autimmene-disease prone Yaa mice. These results indicate that $\mathrm{CD}^{+} 4^{+}$capillaries decreased with endothelial cell injury, evident by their morphological changes. These capillary endothelial cells seemed to play crucial roles in the progression of glomerulonephritis and renal dysfunction. Briefly, several studies have shown an imbalance in endothelial cell proliferation and death that is associated with a decrease in renal capillaries leads to the progression of kidney disease [19, 20, 31-33]. Importantly, hypoxia promotes GLs, similar to mesangial cell proliferation in patients with lupus nephritis and in MRL/MpJ-Fas ${ }^{l p r / l p r}$ mice [34]. Therefore, the loss of $\mathrm{CD} 4^{+}$glomerular capillaries and their morphological changes in Yaa mice occur after the progression of GLs, including the development of membranoproliferative lesions and inflammation.

Furthermore, podocytes are involved in the maintenance of healthy intracapillary environments through crosstalk with glomerular endothelial cells and as a source of VEGF in glomeruli $[35,36]$. Podocyte injury is a critical event that causes albumin hyperfiltration from glomerular capillaries [37]. Indeed, our ultrastructural study showed podocyte foot process effacement in GL model mouse kidneys, but not in those of the TIL model mouse. Thus, pathological changes in the glomerular microenvironment resulting from injury of podocytes and capillary endothelia coordinately aggravate GLs and lead to an elevation in uACR.

Capillaries in the tubulointerstitium are essential for renal oxygen supply and maintenance of kidney tubulointerstitial hemodynamics [38, 39]. TILs activate the endothelium, which may correlate with enhanced inflammation and activation of coagulation that favors further capillary and interstitial injury [40, 41]. Eventually, persistent TILs cause a loss of capillaries in the tubulointerstitium [42]. In the present study, TIL models were created by UUO. The advantage of UUO was that diseased and control kidneys could be obtained from the same mice. TILs model mice clearly showed a decrease in $\mathrm{CD}_{34}{ }^{+}$capillaries in the tubulointerstitium with the progression of TILs, characterized by an increase in infiltrating cells and myofibroblasts, as well as damaged tubules in the tubulointerstitium. Furthermore, the ultrastructural study revealed capillary injuries in TILs in detail. These injuries were characterized by thickened and stratified endothelial cytoplasms with vacuolation, loss of fenestration, detaching endothelia with subendothelial vacuolation, and accumulation of collagen fibers beneath the capillary basement membrane. Thus, 
our results clearly indicate pathological correlations between TILs and capillary injury and/or loss in tubulointerstitium in UUO-based TIL models.

In particular, the number of infiltrating $\mathrm{CD}^{+}$cells strongly correlated with the number of $\mathrm{CD}_{3} 4^{+}$capillaries in TILs of UUO models. Therefore, we presumed that interstitial T-cells mediate inflammation and the accumulation of macrophages in the tubulointerstitium. Importantly, an increase in Iba- $1^{+}$macrophages significantly correlated with a decrease in $\mathrm{CD} 34^{+}$capillaries in the present study. Another study showed that apoptosis of endothelial cells triggered capillary regression by blocking blood flow to the site of apoptosis in macrophage-dependent cell death [43]. Renal fibrosis results from reduced endothelial proliferation following alterations in local expression of both angiogenic and antiangiogenic factors, and this imbalance is mediated by macrophage-associated cytokines, such as interleukin 1 beta, and vasoactive mediators [38]. Based on these findings, we considered tubulointerstitial inflammation, especially macrophage infiltration, to underlie injury of capillary endothelial cells and the subsequent net loss of capillaries.

The present study revealed a significant correlation between the number of $\mathrm{CD} 34^{+}$capillaries and the numbers of IL-1F6/IL-36 $\alpha^{+}$damaged renal tubules and $\alpha \mathrm{SMA}^{+}$ myofibroblasts. Evidently, loss of tubulointerstitial capillaries caused TILs because of local hypoxia, because tubulointerstitial capillaries and renal tubules show functional crosstalk to maintain normal renal interstitial structure and function, including preserving the blood supply to maintain tubular epithelial cells. This speculation is strongly supported by previous study indicating that renal ischemia caused by vascular obliteration is a major contributor to renal fibrosis [3]. Moreover, the tubular epithelium is a source of VEGF in the tubulointerstitium [44]. Therefore, we concluded that injury and/or loss of tubulointerstitial capillaries contribute to the progression of TILs, and fibrosis and tubular damage in particular, in UUO kidneys.

In this study, we examined the decrease in $\mathrm{CD}_{3} 4^{+}$renal capillaries associated with the progression of kidney disease using murine GLs and TILs models. The quantitative changes in $\mathrm{CD}_{34}{ }^{+}$capillaries could represent two possible pathological events: 1) a decrease in capillary number, or 2) a decrease in $\mathrm{CD}_{4} 4^{+}$cell number. As shown in the figures, the numbers of local capillaries decreased in GLs and TILs. However, interestingly, CD $34^{-}$capillaries were found in TILs of UUO models, but not in GLs of Yaa mice. CD34 is a single-pass transmembrane sialomucin protein that is expressed on hematopoietic stem cells and in vascular-associated tissue [45]. It is an important adhesion molecule required for lymphocytes to enter lymph nodes [46]. Kusano et al. suggested that the loss of CD34 ${ }^{+}$ capillaries was a result of the disappearance of cell markers in phenotypically altered endothelium [21]. Therefore, a decrease in $\mathrm{CD} 34^{+}$cells in kidneys indicates a decrease in renal capillaries, as well as altered functions of endothelial cells resulting from decreased CD34 due to capillary injury. In a future study, we will compare the loss of renal capillaries and decreased expression of CD34 as contributors to kidney disease progression. Results of this study indicate the importance of morphology and density of local capillaries in human and animal kidney diseases. Blood vessel has been caught the attention recently as it contribute to stem cell niches in various organs, signifying that vascular system may serve a preserved concerned role for stem cells throughout the body. Moreover, the kidney is a highly vascularized organ, and this study shows that GLs and TILs are associated with reduced number of glomerular capillaries and tubulointerstitial capillaries, respectively. Specially, there is a strong correlations between the number of local capillaries in GLs and TILs and the severity of kidney diseases. Pathological changes might also affect vasculature-associated stem cell niche in the kidney. In the future, we will investigate these possibilities.

\section{Conclusions}

Injury and/or loss of the local renal microvasculature is a prominent feature of kidney disease. Defective microvasculature results from profound infiltration of inflammatory cells and cross-talk with surrounding tissues. The progression of local capillary damage can be considered a doubleedged sword, i.e., both the cause and the effect of kidney disease. Therefore, we conclude that injury and the subsequent loss of local capillaries contributes to the progression of lesions in respective area and kidney dysfunction.

\section{Additional files}

Additional file 1: Outline of the study: Strain and number of mice used in different disease models and protocols. (PDF $24 \mathrm{~kb}$ )

Additional file 2: Glomerular lesion model mice show thickening of glomerular basement membranes (GBMs). (PDF $131 \mathrm{~kb}$ )

Additional file 3: Glomerular histopathology indices in glomerular lesion and tubulointerstitial lesion models. (PDF $18 \mathrm{~kb}$ )

Additional file 4: Tubulointerstitial histopathology indices in glomerular lesion and tubulointerstitial lesion models. (PDF 19 kb)

\section{Abbreviations}

ds-DNA: Double stranded-DNA; g: Gram; GLs: Glomerular lesions; GTA: Glutaraldehyde; IL-1F6: Interleukin 1 family, member 6; NBF: Neutral buffer formalin; PAS-H: Periodic acid-Schiff-hematoxylin; PB: Phosphate buffer;

PBS: Phosphate-buffered saline; PFA: Paraformaldehyde; sBUN: Serum blood urea nitrogen; sCr: Serum creatinine; SE: Standard error; TILs: Tubulointerstitial lesions; UACR: Urinary albumin creatinine ratio; UUO: Unilateral ureteral obstruction; VEGF: Vascular endothelial growth factor; aSMA: Alpha smooth muscle actin

\section{Acknowledgements}

Leading program, Graduate School of Veterinary Medicine, Hokkaido University, Japan. 


\section{Funding}

This research work was supported by a Grant-in-Aid for Scientific Research to a Graduate Student from the Graduate School of Veterinary Medicine, Hokkaido University, Japan (Grant-in Aid AY 2015, PH36150001).

\section{Availability of data and materials}

The corresponding author has all of the raw data. This author can be contacted by email if raw data is needed.

\section{Authors' contributions}

MAM, OI, YHAE, TN, and YK designed and performed the experiments and analyzed these data. MAM, OI and YHAE collected samples. All authors were involved in writing the paper and approved the final manuscript for submission.

\section{Ethics approval}

The authors adhered to the Guide for the Care and Use of Laboratory Animals of Hokkaido University, Graduate School of Veterinary Medicine (approved by the Association for Assessment and Accreditation of Laboratory Animal Care International) and to ARRIVE guidelines. Animal experimentation was approved by the Institutional Animal Care and Use Committee of the Graduate School of Veterinary Medicine, Hokkaido University (approval no. 13-0032, 16-0124).

\section{Consent for publication}

Not applicable.

\section{Competing interests}

The authors declare that they have no competing interests.

\section{Publisher's Note}

Springer Nature remains neutral with regard to jurisdictional claims in published maps and institutional affiliations.

\section{Author details}

${ }^{1}$ Laboratory of Anatomy, Graduate School of Veterinary Medicine, Hokkaido University, Kita 18, Nishi 9, Kita-ku, Sapporo, Japan. ²Department of Histology, Faculty of Veterinary Medicine, Zagazig University, Zagazig, Egypt. ${ }^{3}$ Section of Biological Safety Research, Chitose Laboratory, Japan Food Research Laboratories, Tokyo, Japan.

\section{Received: 16 January 2017 Accepted: 21 August 2017}

\section{Published online: 04 September 2017}

\section{References}

1. Tonelli M, Wiebe N, Culleton B, House A, Rabbat C, Fok M, McAlister F, et al. Chronic kidney disease and mortality risk: a systematic review. J Am Soc Nephrol. 2006;17:2034-47.

2. Ohashi R, Kitamura H, Yamanaka N. Peritubular capillary injury during the progression of experimental glomerulonephritis in rats. J Am Soc Nephrol. 2000;11(1):47-56.

3. Fine LG, Norman JT. Chronic hypoxia as a mechanism of progression of chronic kidney diseases: from hypothesis to novel therapeutics. Kidney Int. 2008;74:867-72.

4. Mayer G. Capillary rarefaction, hypoxia, VEGF and angiogenesis in chronic renal disease. Nephrol Dial Trans. 2011;26:1132-7.

5. Nakagawa T, Lan HY, Zhu HJ, Kang DH, Schreiner GF, Johnson RJ. Differential regulation of VEGF by TGF-beta and hypoxia in rat proximal tubular cells. Am J Physiol Renal Physiol. 2004;287:F658-64.

6. Olszewska-Pazdrak B, Hein TW, Olszewska P, Carney DH. Chronic hypoxia attenuates VEGF signaling and angiogenic responses by downregulation of KDR in human endothelial cells. Am J Physiol Cell Physiol. 2009:296:C1162-70.

7. Endemann DH, Schiffrin EL. Endothelial dysfunction. J Am Soc Nephrol. 2004;15:1983-92.

8. Cheng H, Harris RC. Renal endothelial dysfunction in diabetic nephropathy. Cardiovasc Hematol Disord Drug Targ. 2014;14:22-33.

9. Tang $R$, Han $Y$, Wu M, Zhu D, Liu B. The effects of endothelial injury in renal fibrosis progression. Aust J Nephrol Hypertens. 2014;1 (5):1021.

10. Advani A, Gilbert RE. The endothelium in diabetic nephropathy. Semin Nephrol. 2012;32:199-207.

11. Panzer U, Steinmetz OM, Reinking RR, Meyer TN, Fehr S, Schneider A, et al. Compartment-specific expression and function of the chemokine IP-10/
CXCL10 in a model of renal endothelial microvascular injury. J Am Soc Nephrol. 2006;17:454-64

12. Lim YC, Garcia-Cardena G, Allport JR, Zervoglos M, Connolly AJ, Gimbrone MA Jr, et al. Heterogeneity of endothelial cells from different organ sites in T-cell subset recruitment. Am J Pathol. 2003;162:1591-601.

13. Lindenmeyer MT, Kretzler M, Boucherot A, Berra S, Yasuda Y, Henger A, et al. Interstitial vascular rarefaction and reduced VEGF-A expression in human diabetic nephropathy. J Am Soc Nephrol. 2007;18:1765-76.

14. Kimura J, Ichii O, Nakamura T, Horino T, Otsuka S, Kon Y. BXSB-type genome causes murine autoimmune glomerulonephritis: pathological correlation between telomeric region of chromosome 1 and Yaa. Genes Immun. 2014;15:182-9.

15. Kelley VE, Roths JB. Interaction of mutant Ipr gene with background strain influences renal disease. Clin Immu Immunopath. 1985:37:220-9.

16. Kimura J, Ichii O, Otsuka S, Kanazawa T, Namiki Y, Hashimoto Y, Kon Y. Quantitative and qualitative urinary cellular patterns correlate with progression of murine glomerulonephritis. PLoS One. 2011;6:e16472.

17. Vaughan ED, Marion D, Poppas DP, Felsen D. Pathophysiology of unilateral ureteral obstruction: studies from Charlottesville to New York. J Urol. 2004;172:2563-9.

18. Ridson RA, Sloper JC, De Wardener HE. Relationship between renal function and histological changes found in renal biopsy specimens from patients with persistent glomerular nephritis. Lancet. 1968;2(7564):363-6.

19. Takahashi T, Huynh-Do U, Daniel TO. Renal microvascular assembly and repair: power and promise of molecular definition. Kidney Int. 1998;53:826-35.

20. Shimizu A, Kitamura H, Masuda Y, Ishizaki M, Suqisaki Y, Yamanaka N. Rare glomerular capillary regeneration and subsequent capillary regression with endothelial cell apoptosis in progressive glomerulonephritis. Am J Path. 1997;151(5):1231-9.

21. Kusano T, Takano H, Kang D, Nagahama K, Aoki M, Morita M, et al. Endothelial cell injury in acute and chronic glomerular lesions in patients with IgA nephropathy. Human Pathol. 2016:49:135-44.

22. Ichii O, Otsuka S, Sasaki N, Yabuki A, Ohta H, Takiguchi M, et al. Local overexpression of interleukin-1 family, member 6 relates to the development of tubulointerstitial lesions. Lab Investig. 2010;90:459-75.

23. Ichii O, Konno A, Sasaki N, Endoh D, Hashimoto Y, Kon Y. Autoimmune glomerulonephritis induced in congenic mouse strain carrying telomeric region of chromosome 1 derived from MRL/MpJ. Histol Histopathol. 2008:23:411-22.

24. Verli FD, Rossi-Schneider TR, Schneider FL, Yurgel LS, de Souza MA. Vascular corrosion casting technique steps. Scanning. 2007;29:128-32.

25. Walker EJ, Shen F, Young WL, Su H. Cerebrovascular casting of the adult mouse for 3D imaging and morphological analysis. J Vis Exp. 2011; doi:10.3791/2958.

26. Nowling TK, Gilkeson GS. Mechanisms of tissue injury in lupus nephritis. Arthritis Res Ther. 2011; doi:10.1186/ar3528.

27. Ichii O, Konno A, Sasaki N, Endoh D, Hashimoto Y, Kon Y. Altered balance of inhibitory and active Fc gamma receptors in murine autoimmune glomerulonephritis. Kidney Int. 2008;74:339-47.

28. Bekar KW, Owen T, Dunn R, Ichikawa T, Wang W, Wang R, et al. Prolonged effects of short-term anti-CD20 B cell depletion therapy in murine systemic lupus erythematosus. Arthr Rheum. 2010;62:2443-57.

29. Liapis G, Singh HK, Derebail VK, Gasim AM, Kozlowski T, Nickeleit V. Diagnostic significance of peritubular capillary basement membrane multilaminations in kidney allografts: old concepts revisited. Transplantation. 2012:94(6):620-9.

30. de Kort H, Moran L, Roufosse C. The role of electron microscopy in renal allograft biopsy evaluation. Curr Opin Organ Transplant. 2015:20(3):333-42.

31. Yamanaka N, Shimizu A. Role of glomerular endothelial damage in progressive renal disease. Kid Blood Press Res. 1999;22:13-20.

32. Kitamura H, Shimizu A, Masuda Y, Ishizaki M, Sugisaki Y, Yamanaka N. Apoptosis in glomerular endothelial cells during the development of glomerulosclerosis in the remnant kidney model. Exp Nephrol. 1998;6:328-36.

33. Cook HT, Pickering MC. Histopathology of MPGN and C3 glomerulopathies. Nat Rev Nephrol. 2015;11:14-22.

34. Deng W, Ren $Y$, Feng $X$, Yao G, Chen W, Sun Y, et al. Hypoxia inducible factor-1 alpha promotes mesangial cell proliferation in lupus nephritis. Am J Nephrol. 2014;40:507-15.

35. Kobayashi N, Ueno T, Ohashi K, Yamashita H, Takahashi $Y$, Sakamoto K, et al. Podocyte injury-driven intracapillary plasminogen activator inhibitor type 1 accelerates podocyte loss via uPAR-mediated $\beta 1$-integrin endocytosis. Am J Physiol Renal Physiol. 2015;308:F614-26.

36. Nihalani D, Susztak K. Sirt1-Claudin-1 crosstalk regulates renal function. Nat Med. 2013;19:1371-82. 
37. Kimura J, Ichii O, Otsuka S, Sasaki H, Hashimoto Y, Kon Y. Close relations between podocyte injuries and membranous proliferative glomerulonephritis in autoimmune murine models. Am J Nephrol. 2013;38:27-38.

38. Kang DH, Kanellis J, Hugo H, Truong L, Anderson S, Kerjaschki D, et al. Role of the microvascular endothelium in progressive renal disease. J Am Soc Nephrol. 2002;13:806-16.

39. Nangaku M. Mechanisms of tubulointerstitial injury in the kidney: final common pathways to end-stage renal failure. Intern Med. 2004;43:9-17.

40. Ishidoya S, Morrisey J, McCracken R, Klahr S. Delayed treatment with enalapril halts tubulointerstitial fibrosis in rats with obstructive nephropathy. Kidney Int. 1996:49:1110-9.

41. Pichler RH, Franceschini N, Young BA, Hugo C, Andoh TF, Burdmann EA, et al. Pathogenesis of cyclosporine nephropathy: roles of angiotensin II and osteopontin. J Am Soc Nephrol. 1995;6(4):1186-96.

42. Nicolic-Paterson DJ, Lan HY, Atkins RC. Macrophages in renal injury. Kidney Int Suppl. 1994;45:S79-82.

43. Meeson A, Palmer M, Calfon M, Lang R. A relationship between apoptosis and flow during programmed capillary regression is revealed by vital analysis. Development. 1996;122(12):3929-38.

44. Schrijvers BF, Flyvbjerg De Vriese AS. The role of vascular endothelial growth factor (VEGF) in renal pathophysiology. Kidney Int. 2004:2003-17.

45. Nielsen JS, McNagny KM. Novel functions of the CD34 family. J Cell Sci. 2008;121:3683-92.

46. Berg EL, Mullowney AT, Andrew DP, Goldberg JE, Butcher EC. Complexity and differential expression of carbohydrate epitopes associated with Lselectin recognition of high endothelial venules. Am J Pathol. 1998 Feb; 152(2):469-77

\section{Submit your next manuscript to BioMed Central and we will help you at every step:}

- We accept pre-submission inquiries

- Our selector tool helps you to find the most relevant journal

- We provide round the clock customer support

- Convenient online submission

- Thorough peer review

- Inclusion in PubMed and all major indexing services

- Maximum visibility for your research

Submit your manuscript at www.biomedcentral.com/submit 